\title{
On stringy de Sitter spacetimes
}

\section{Per Berglund, ${ }^{a}$ Tristan Hübsch ${ }^{b}$ and Djordje Minićc}

${ }^{a}$ Department of Physics and Astronomy, University of New Hampshire, 9 Library Way, Durham, NH 03824, U.S.A.

${ }^{b}$ Department of Physics and Astronomy, Howard University, 2355 Sixth Street, NW, Washington, DC 20059, U.S.A.

${ }^{c}$ Department of Physics, Virginia Tech, 850 West Campus Dr., Blacksburg, VA 24061, U.S.A.

E-mail: per.berglund@unh.edu, thubsch@howard.edu, dminic@vt.edu

ABSTRACT: We reexamine a family of models with a 3+1-dimensional de Sitter spacetime obtained in the standard tree-level low-energy limit of string theory with a non-trivial anisotropic axion-dilaton background. While such limiting approximations are encouraging but incomplete, our analysis reveals a host of novel features, and shows these models to relate standard and well understood supersymmetric string theory solutions. Finally, we conjecture that this de Sitter spacetime naturally arises by including more of the stringy degrees of freedom, such as a recently advanced variant of the non-commutative phasespace formalism, as well as the analytic continuation of a complex two-dimensional Fano variety arising as a small resolution in a Calabi-Yau 5-fold.

KEYwords: F-Theory, String theory and cosmic strings

ARXIV EPRINT: 1902.08617 


\section{Contents}

1 Introduction, results and synopsis 1

2 Non-holomorphic deformations in F-theory 2

2.1 A roadmap 3

2.2 Phenomenology 5

3 The main point $\quad 7$

3.1 Stability 8

4 Novel features and consequences $\quad 10$

$\begin{array}{lll}4.1 \text { Anisotropy } & 11\end{array}$

$\begin{array}{lll}4.2 & \text { Holomorphic limits } & 12\end{array}$

5 Generalizations and implications $\quad 13$

$\begin{array}{ll}5.1 \text { Seeing double } & 14\end{array}$

$\begin{array}{ll}5.2 \text { More on stability } & 16\end{array}$

6 Summary, outlook and conclusions $\quad 16$

\section{Introduction, results and synopsis}

Whether or not asymptotically de Sitter spacetime can exist as a solution of string theory has been one of the fundamental conundrums in string theory ever since the dramatic discovery of dark energy in the late 1990s [1, 2]. This question is still considered open [3, 4], and the interest in this hard and fundamental issue has been reignited recently $[5,6]$.

Within the standard low-energy limit of string theory, we focus on the effective action for Einstein's gravity (we concentrate on the observed 4-dimensional case) in the familiar format (we adopt the "mostly positive" metric signature throughout):

$$
S_{\text {eff }}=\int d^{4} x \sqrt{g}\left(-\frac{1}{8 \pi G} \Lambda+\frac{1}{16 \pi G} R+a R_{\mu \nu} R^{\mu \nu}+b R^{2}+c R_{\mu \nu \rho \sigma} R^{\mu \nu \rho \sigma}+\ldots\right) .
$$

The coefficients $a, b, c$ as well as the omitted metric/curvature terms are completely determined by the renormalization of the underlying worldsheet theory $[7,8]$, and the usual formulation of (target) spacetime in string theory, identified with the vev's of certain "coordinate" quantum fields in the underlying worldsheet field theory [9-11]. Also omitted from (1.1) are "matter" terms, the relevant of which are discussed below. 
In particular, we will focus on a class of models arising from type IIB/F-theory, the so called 'axilaton' models ${ }^{1}$ [12-17]: (1) evade the oft-mentioned no-go theorem ${ }^{2}$ [19-22], (2) form a discretuum owing to their stringy $\mathrm{SL}(2 ; \mathbb{Z})$ monodromy, $(3)$ the overall lengthscale $\ell$ is determined by dimensional transmutation, (4) require $g_{s} \sim O(1)$, with an effective incorporation of S-duality, and (5) relate two classes of standard supersymmetric string theory solutions [23, 24] and [25], and a third, novel class. This resonates with some recent assessments [26], and some features of the recent efforts [27, 28]; it reminds of the "T-fold" constructions [29-32], and qualifies the standard low-energy effective theory limit description as encouraging but incomplete: more of the stringy degrees of freedom must be included, as also advocated recently in the phase-space approach [33-41], and also in the different, earlier double field theory approach [42-46]. These characteristics jointly imply that a more accurate description of de Sitter spacetime in string theory can only be achieved beyond the standard low-energy effective field theory limit.

Section 2 reviews the 'axilaton' models as an iterative deformation of Minkowski supersymmetric string (and F-)theory compactifications into de Sitter solutions with cosmologically broken supersymmetry. Focusing on the 'axilaton' background in the F-theory formulation of type-IIB string theory [23], we show in section 3 that the above-listed properties (2)-(3) imply the various parameters in these models to be stable. The key results (4)-(5) are discussed in section 4 .

In section 5 , we discuss analogous constructions driven by the dynamics of compactification moduli, which then resemble the T-folds [29-32] wherein (T-dual) mirror-symmetry is involved in patching local charts. The 'axilaton' models involve S-duality in a similar vein, so that these two construction types combine easily and define a very general class of models. Also, the Minkowski $\rightarrow$ de Sitter metric deformation correlates with the desingularization of the total spacetime in 'axilaton' models, implying (if tentatively) that 3+1-dimensional de Sitter spacetime occurs generically within string theory, via so-called exceptional subspaces [47].

\section{Non-holomorphic deformations in F-theory}

Our 'axilaton' models are a deformation of the stringy cosmic string, and as such are effective stringy solutions [48]. They are naturally equipped with the generalized geometric (and non-commutatively doubled) spacetime structure discussed recently in [33-41], which is a unique characteristic of (even effective) string theory. As we will show, no such generalized geometric stringy solution has moduli: each is stabile by virtue of a (Coulomb/QCD-like) "dimensional transmutation" mechanism. We emphasize that this is not the usual mechanism for understanding the emergence of (metastable) de Sitter space in string theory, where one invokes an effective potential that explicitly shows the possible emergence of

\footnotetext{
${ }^{1}$ The admittedly playful name is a reminder that the deformation family of the spacetime varying string vacua of refs. [12-17] are driven by the background values of the axion-dilaton system and their axial $\mathrm{SL}(2 ; \mathbb{Z})$ monodromy in a transversal 2-dimensional plane $\mathscr{Y}^{2}$, around the non-compact spacetime, $\mathscr{W}$. The succinct name also saves us from repeated circumlocutions that perforce include this string of references.

${ }^{2}$ For other examples that evade the no-go theorem, see [18].
} 
a moduli-free metastable de Sitter solution. (A good example of a generalized geometric solution with such features is based on asymmmetric orientifolds, as discussed in $[49,50])$. However, the non-commutatively doubled spacetime structure of our solution allows for a dual interpretation, in analogy with the discussion of confinement in QCD in terms of dual, magnetic, variables [51, 52]: this enables, in principle, a construction of an effective potential and the canonical discussion of moduli stabilization in terms of such an effective potential, written however in terms of dual spacetime variables; we defer this to a separate study.

In particular, the 'axilaton' models were developed [12-17] as an iterated, non-holomorphic deformation (and partial decompactification) of standard string compactifications, focusing for simplicity on the special case driven by the axion-dilaton system in the F-theory description of type-IIB string theory [23]. These models turn out to relate well-known Ftheory configurations, imply a dependence on additional stringy degrees of freedom, and are related to the recently uncovered non-commutatively doubled phase-space formulation of string theory [33-41]. Furthermore, this sets the stage for an improved understanding of the resulting de Sitter spacetimes, to which we will turn in the latter sections of the paper. First however, we provide a roadmap to this iterative and ultimately non-holomorphic deformation and specify the notation, setting the stage for deriving and discussing the above-itemized key results in sections 3 and 4, and generalizations in section 5 .

\section{$2.1 \quad$ A roadmap}

The 'axilaton' deformation family of models is constructed by starting with an F-theoretic type-IIB string theory spacetime, $\mathscr{W}^{3,1} \times \mathscr{Y}^{6}\left(\times T^{2}\right)$, where the complex structure of the zero-size "hidden" $T^{2}$ fiber of F-theory is identified with the axion-dilaton $\tau \stackrel{\text { def }}{=} \alpha+i e^{-\Phi}$ modulus [23]. ${ }^{3}$

1. Deform this à la stringy cosmic strings $[53,54]$ into $^{4} \mathscr{W}^{3,1} \rtimes \mathscr{Y}^{2} \times \mathscr{Y}^{4}$, where:

(a) $\tau$ and the observable spacetime $\mathscr{W}^{3,1}$ (via warped metric) vary over $\mathscr{Y}^{2}$,

(b) $\mathscr{Y}^{2} \rightarrow S^{1} \times \mathscr{Z}$, with the polar parametrization $r e^{i \theta}=\ell e^{z+i \theta}$,

(c) $\mathscr{Y}^{4}=\mathrm{K} 3$ or $T^{4}$ preserves supersymmetry. ${ }^{5}$

While $\tau$ (and the "hidden" $T^{2}$ ) is holomorphic over $\mathscr{Y}^{2} \approx \mathbb{C}^{1}=\left(\mathbb{P}^{1} \backslash\{\infty\}\right), \mathscr{W}_{\left(z_{i}, \theta_{i}\right)}^{3,1}$ are cosmic 3 -branes at special isolated points $\left(z_{i}, \theta_{i}\right) \in \mathscr{Y}^{2}[53,54]$.

2. Deform $\tau$ to vary non-holomorphically, only over $S^{1} \subset \mathscr{Y}^{2}$, while the metric varies only over $\mathscr{Z} \subset \mathscr{Y}^{2},-$ and turns complex beyond $z_{0}$, which locates the circular naked singularity. As the proper distance to both ends of $z \in \mathscr{Z}=\left(-\operatorname{sgn}\left(z_{0}\right) \cdot \infty, z_{0}\right)$ is infinite, $\mathscr{Z} \approx \mathbb{R}^{1}$ always: $\mathscr{Y}^{2} \approx \mathbb{C}^{1}$ has been punctured into $S^{1} \times \mathscr{Z}$ and the cosmic branes of step 1 in the above roadmap have effectively coalesced to $z \rightarrow z_{0}$ and $-\operatorname{sgn}\left(z_{0}\right) \cdot \infty$.

3. Cross-patching the two distinct solutions, $\mathscr{Z}_{+}=\left(-\infty, z_{0}\right]$ for $z_{0}>0$ and $\mathscr{Z}_{-}=\left[z_{0}, \infty\right)$ for $z_{0}<0$, at $z=0$ into two annuli/cylinders: one with the naked singularity at the ends of $\mathscr{Z} \approx \mathbb{R}^{1}$ and one without, but both with extra, $\delta(z)$-localized matter required by matching conditions [13].

\footnotetext{
${ }^{3}$ As usual, only the complex structure of the $T^{2}$ is relevant, with the volume having been shrunk to zero.

${ }^{4}$ Here, we borrow the group-theoretic symbol " $\rtimes$ " to denote that $\mathscr{W}^{3,1}$ varies (is fibered) over $\mathscr{Y}^{2}$.

${ }^{5}$ It is straightforward to also fiber $\mathscr{Y}^{4}$ over $\mathscr{Y}^{2}$, thus relating the 'axilaton' models to the virtually ubiquitous K3- and elliptic fibration models; see section 5 .
} 

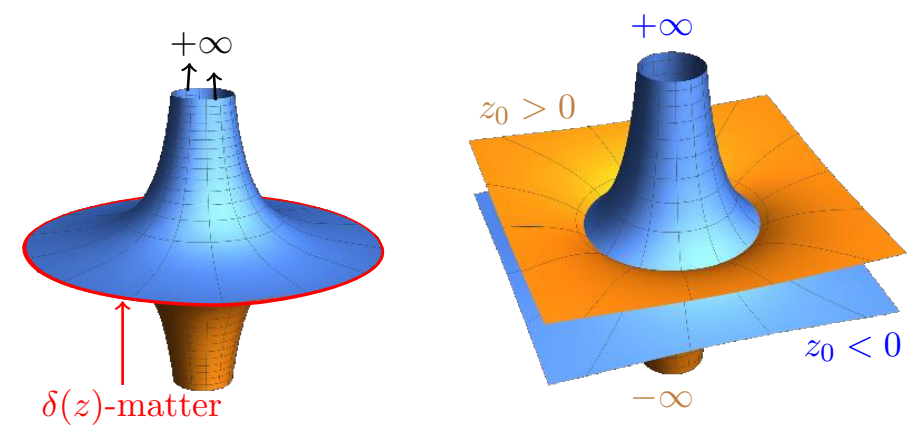

naked singularity at $z_{0}>0$

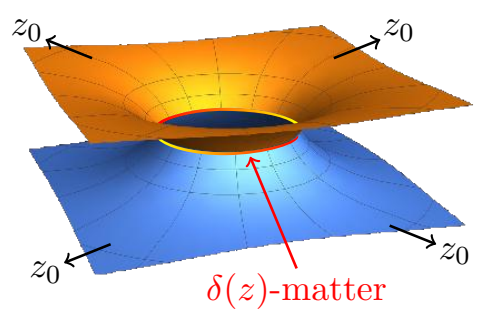

naked singularity at $z_{0}<0$

Figure 1. Plotting (vertically) the proper distance against (radially) the circumference in the horizontal plane; the two simple solutions (center), patched solutions (left and right).

4. Deforming the $\mathscr{W}_{z=0}^{3,1}$ metric to de Sitter $\left(\Lambda_{b}>0\right)$ removes the spacetime curvature singularities at $z_{0} \in \mathscr{Z}$ of the Minkowski solution $[12,13]$.

As noted in steps 2 and 3 of the above roadmap, proper distance to both ends of $z \in$ $\left(-\operatorname{sgn}\left(z_{0}\right) \infty, z_{0}\right)$ is infinite, as is the proper circumference at $z_{0}$; see figure 1 . Therefore, all versions of $\mathscr{Y}^{2}$ in the 'axilaton' configurations are diffeomorphic to annuli/cylinders, which are non-compact Calabi-Yau 1-folds.

Warping. While $D=6$ is the phenomenologically relevant case, where $\mathscr{W}_{z=0}^{3,1}$ is $D-2=$ $3+1$-dimensional, working in a general $D$-dimensional background highlights the inherent $D$-dependence. Having fixed $\mathscr{Y}^{4}=\mathrm{K} 3$ or $T^{4}$, we omit this factor until section 5 .

The codimension-2 solution $\mathscr{W}^{3,1} \rtimes\left(S^{1} \times \mathscr{Z}\right)$ of the final step 4 in the above roadmap, has a positive cosmological constant, $\Lambda_{b}$, along $\mathscr{W}^{3,1}$, and the warped metric is:

$$
\begin{aligned}
\mathrm{d} s^{2} & =A^{2}(z) \bar{g}_{a b} \mathrm{~d} x^{a} \mathrm{~d} x^{b}+\ell^{2} B^{2}(z)\left(\mathrm{d} z^{2}+\mathrm{d} \theta^{2}\right), \\
\bar{g}_{a b} \mathrm{~d} x^{a} \mathrm{~d} x^{b} & =-\mathrm{d} x_{0}^{2}+e^{2 \sqrt{\Lambda_{b}} x_{0}}\left(\mathrm{~d} x_{1}^{2}+\ldots+\mathrm{d} x_{D-3}^{2}\right),
\end{aligned}
$$

where $z:=\log (r / \ell) \in \mathscr{Z}[16]$.

The modulus. The two explicit solutions for $\tau$ satisfying the above separation of variables [12, 13]:

$$
\begin{aligned}
\tau_{I}(\theta) & =b_{0}+i g_{s}^{-1} e^{\omega\left(\theta-\theta_{0}\right)} \\
\tau_{I I}(\theta) & =\left(b_{0} \pm g_{s}^{-1} \tanh \left[\omega\left(\theta-\theta_{0}\right)\right]\right) \pm i g_{s}^{-1} \operatorname{sech}\left[\omega\left(\theta-\theta_{0}\right)\right]
\end{aligned}
$$

are anisotropic and aperiodic over $|\theta| \leq \pi$, but exhibit a non-trivial SL $(2 ; \mathbb{Z})$ monodromy for specific choices in the effective parameter space $\left(b_{0}, \omega, g_{s}\right)$; the $\theta_{0}$-dependence in (2.2) may be absorbed by suitably redefining $g_{s}$ [12]. With the Teichmüller metric $\mathcal{G}_{\tau \bar{\tau}}=-1 /(\tau-\bar{\tau})^{2}$, the $\mathcal{G}_{\tau \bar{\tau}}|\partial \tau|^{2}$ addition to the action (1.1) is $\mathrm{SL}(2 ; \mathbb{Z})$-invariant.

The metric. With the metric (2.1a) axially symmetric while $\tau$ is independent of the radial distance from the cosmic brane (2.2), the Einstein equation simplifies to:

$$
R_{\mu \nu}=\mathcal{G}_{\tau \bar{\tau}} \partial_{\mu} \tau \partial_{\nu} \bar{\tau} \stackrel{\text { def }}{=} \widetilde{T}_{\mu \nu}=\operatorname{diag}\left[0, \cdots, 0,(\omega / 2 \ell)^{2}\right], \quad \text { with } \mathcal{G}_{\tau \bar{\tau}}=-\frac{1}{(\tau-\bar{\tau})^{2}}
$$


Thereby, although the total spacetime $\mathscr{W}^{3,1} \rtimes \mathscr{Y}^{2} \times \mathscr{Y}^{4}\left(\times T^{2}\right)$ in fact does admit a Ricciflat metric, the background 'axilaton' configuration (2.2) drives the spacetime metric to deform away from the Ricci-flat choice. This deviation from Ricci-flatness is clearly characterized (2.3) by the anisotropy $\omega \in \mathbb{R}$ (so $\omega^{2}>0$ ) that induces supersymmetry breaking [12], and by the characteristic (transversal) length scale $\ell$ in $\mathscr{Y}^{2}$.

For $\omega \neq 0(\tau \neq$ const. $)$, one has a perturbative, analytic solution: ${ }^{6}$

$$
\begin{aligned}
& A(z)=Z(z)\left(1-\frac{\omega^{2} z_{0}^{2}(D-3)}{24(D-1)(D-2)} Z(z)^{2}+O\left(\omega^{4}\right)\right), \\
& B(z)=\frac{1}{\ell z_{0} \sqrt{\Lambda_{b}}}\left(1-\frac{\omega^{2} z_{0}^{2}}{8(D-1)} Z(z)^{2}+O\left(\omega^{4}\right)\right),
\end{aligned}
$$

where $Z(z) \stackrel{\text { def }}{=} 1-z / z_{0}$ for $z_{0}>0$. Spacetime is asymptotically flat approaching the horizon $z=z_{0}$ (at infinite proper distance) [16], in agreement with the behavior of Rindler space [57].

In sharp contrast, the $\Lambda_{b}=0$ Minkowski solution $[12,13]$ is radically different:

$$
\tilde{A}(z)=Z(z)^{\frac{1}{(D-2)}}, \quad \tilde{B}(z)=Z(z)^{-\frac{(D-3)}{2(D-2)}} e^{-\frac{1}{2}(Z(z)-1)[\xi(Z(z)+1)+2]},
$$

and exhibits a naked singularity at $z=z_{0}$, beyond which the metric becomes complex. The parameter $\xi$ counts cosmic branes in units of $\pm \frac{1}{12}$ [53], and was set to $\xi=-1$ in ref. [16]. The radical difference between the $\Lambda_{b} \neq 0$ de Sitter solutions (2.4) and the $\Lambda_{b}=0$ Minkowski solution (2.5) indicates that this is not at all a straightforward $\Lambda_{b} \rightarrow 0$ limit, reminding of the radical metric changes in the "blowup" surgery [58].

\subsection{Phenomenology}

General case. Whereas $\Lambda_{b}>0$ (i.e., $\omega^{2}>0$ ) desingularizes the metric (2.1) with (2.4), eq. (2.5) is still a good approximation to eq. (2.4) away from $z_{0} \cdot{ }^{7}$ In particular, by comparing eq. (2.4) with eq. (2.5) close to the core one can show that $[12,16]$ :

$$
\begin{aligned}
& z_{0}=-\left.\frac{h}{h^{\prime}}\right|_{z=0}, \quad \xi=\left.\left(\frac{h^{\prime \prime}}{2 h^{\prime}}-\frac{\omega^{2} h}{8 h^{\prime}}\right)\right|_{z=0}, \quad \omega_{0}^{2} \stackrel{\text { def }}{=} 8 \xi / z_{0}, \\
& \ell=\left.\Lambda_{b}^{-1 / 2} \sqrt{\frac{h^{\prime \prime} h^{-\frac{(D-4)}{(D-2)}}}{(D-2)(D-3)}}\right|_{z=0}, \quad h(z) \stackrel{\text { def }}{=} A(z)^{D-2}=\left(1-z / z_{0}\right)^{D-2} .
\end{aligned}
$$

That is, given a smooth solution defined by (2.4) and parameterized in terms of $\left(z_{0}, \omega, \Lambda_{b}\right)$, this solution close to $z=0$ can be interpreted as a cosmic brane solution with parameters $\left(z_{0}, \xi, \ell\right)$ determined by the $\left(z_{0}, \omega, \Lambda_{b}\right)$ through eqs. (2.6). Alternatively, we can solve for $\Lambda_{b}$,

$$
\Lambda_{b}=\frac{\left(\omega^{2}-\left.\omega_{0}^{2} A^{2}\right|_{z=0}\right)}{4 \ell^{2}(D-2)(D-3)} \stackrel{\text { def }}{=} \frac{\Delta \omega^{2}}{4 \ell^{2}(D-2)(D-3)} .
$$

The anisotropy, $\omega$, determines the stress tensor (2.3) for the de Sitter $\left(\Lambda_{b}>0\right)$ solution, and approaches the anisotropy, $\omega_{0}$, at $z \rightarrow 0$, of the Minkowski $\left(\Lambda_{b}=0\right)$ cosmic brane solution. The cosmological constant is thus directly related to the non-trivial variation of the

\footnotetext{
${ }^{6}$ This solution is of the same form as that discussed by Gregory [55, 56] for the U(1) vortex solution.

${ }^{7}$ This was first shown by Gregory $[55,56]$ and later realized in the present context in ref. [16].
} 
modulus $\tau$ — and thereby the string coupling constant — as a function of $\theta$ ! This gives a very non-trivial relation between the stringy moduli, and hence string theory itself, and a positive $\Lambda_{b}$. Furthermore, $\Lambda_{b}>0$ is equivalent ${ }^{8}$ to $\omega^{2}>\omega_{0}^{2}$, so that $\omega^{2}=0$ also implies that $\omega_{0}^{2}=0$. The latter being a necessary condition for restoring supersymmetry establishes the important relation between supersymmetry breaking and a positive cosmological constant.

Finally, the Newton constant, $G_{N}^{(D-2)}=M_{D-2}^{-(D-4)}$, in $D-2$ dimensions and the volume of the transverse space, $\mathscr{Y}^{2}$, are [16]:

$$
G_{N}^{(D-2)}=M_{D}^{-(D-2)} \operatorname{Vol}\left(\mathscr{Y}^{2}\right)^{-1}, \quad \text { and } \quad \operatorname{Vol}\left(\mathscr{Y}^{2}\right) \sim \frac{\pi}{D-3} \frac{\ell}{\sqrt{\Lambda_{b}}} .
$$

The volume of the transverse space, $\operatorname{Vol}\left(\mathscr{Y}^{2}\right)$, has been explicitly shown to be finite but large in spite of its non-compactness and the infinite proper distance to $z_{0}$ [16]; see also eq. (3.3). This factor drives the exponentially large $M_{D-2} / M_{D}$ hierarchy, and was then shown [16] to imply the following relation,

$$
\Lambda_{D-2} \sim\left(\frac{\pi}{D-3}\right)^{2} M_{D-2}^{D-2}\left(\ell M_{D-2}\right)^{2}\left(\frac{M_{D}}{M_{D-2}}\right)^{2 D-4}
$$

where $\Lambda_{D-2}=\Lambda_{b} / G_{N}^{(D-2)}$ is the energy density in $D-2$ dimensions.

Four dimensions. Employing the phenomenologically relevant case of $D=6$, recall that $\ell$ is the characteristic $\operatorname{Vol}\left(\mathscr{Y}^{2}\right)$-related size of the cosmic brane. Whereas no concrete physical mechanism is known to determine this scale, we will return to this question below. However, should $\ell$ be stabilized by a longitudinal 4-dimensional physics mechanism, ${ }^{9}$ then $\ell \sim M_{4}^{-1}$ and (up to factors of $\mathcal{O}(1)$ )

$$
\Lambda_{4} \sim M_{4}{ }^{4}\left(\frac{M_{6}}{M_{4}}\right)^{8}
$$

The original scenario of ref. [16] with $D=6$ then applies, wherein the 10-dimensional spacetime of the Type IIB string theory was first compactified on a 4-dimensional supersymmetry preserving space, ${ }^{10} \mathscr{Y}^{4}$, of characteristic size $M_{10}^{-1}=M_{6}^{-1} \sim(10 \mathrm{TeV})^{-1} \sim 10^{-19} \mathrm{~m}$. The cosmic brane of ref. [16] then describes a 3+1-dimensional de Sitter spacetime, with the characteristic scale $M_{4} \sim 10^{19} \mathrm{GeV}$. Furthermore, $L \stackrel{\text { def }}{=} \Lambda_{b}^{-1 / 2} \sim 10^{41} \mathrm{GeV}^{-1} \sim 10^{25} \mathrm{~m}$, provides a natural scale which coincides with the Hubble radius.

Conversely, although the generic 'axilaton' models break supersymmetry, their parameter space contains the $\omega \rightarrow 0$ supersymmetric and well understood limits [23, 24] and [25] (see (4.7), below), wherein

$$
\left(\Lambda_{b} \sim M_{P}^{4}\left(M_{\text {susy }} / M_{P}\right)^{8}\right) \rightarrow 0, \quad \text { i.e., } \quad \Lambda_{b}, M_{\text {susy }} \rightarrow 0 .
$$

The 'axilaton' deformation family of models are thus explicitly constructed as supersymmetry-breaking and positive (de Sitter) cosmological constant inducing deformations of the familiar supersymmetric (and Minkowski) configurations in F-theory.

\footnotetext{
${ }^{8}$ That $\Lambda_{b}$ must be positive follows directly from the defining equation for the warp factor $A(z)[16]$.

${ }^{9}$ There exist both field and string theory arguments of this type [59-61]; see also below.

${ }^{10}$ All remaining supersymmetry will be broken by the cosmic brane solution [12].
} 


\section{The main point}

Given the above properties of our solution, the main new point we want to make regarding the existence of de Sitter backgrounds in string theory is as follows: string theory has purely stringy degrees of freedom (for example, the difference between the left and right string modes) not captured by the usual effective field theory/spacetime description used in the standard discussions, based on string compactifications, regarding the problem of de Sitter space in string theory [62-64]. Such purely stringy (and, in general, chiral and non-commutative) degrees of freedom are captured in a non-commutative phase-space formulation of string theory [33-41]. In that formulation, we can integrate over such dual/"momentum" stringy degrees of freedom in order to generate an effective spacetime description. This differs from the usual effective field theory approach and reproduces to lowest order the sequestering mechanism [65], as reported elsewhere [66]. Our proposal is that this procedure will naturally lead to an effective de Sitter background, by inducing an effective dilaton (captured by the above $\omega \theta$-dependence) background which corresponds to the dilaton profile of our solution. This effective dilaton profile is anisotropic, and it is precisely the degree of anisotropy, $\omega$, that generates a positive cosmological constant of our solution.

The resulting de Sitter background can be understood as a geometric deformation relating different well understood supersymmetric backgrounds of F-theory. (In addition, as we argue below, the resulting de Sitter space can be also understood as a blow-up resolution of a singular Minkowski limit.) The above see-saw like relation between the cosmological constant scale and the scale of gravity and the scale of particle physics/supersymmetry breaking, is set by the requirements of a stringy $\mathrm{SL}(2 ; \mathbb{Z})$ monodromy (as we shall argue in what follows) and in particular the S-duality part of it, by viewing our solution as an $S$-fold ${ }^{11}$ in the context of the recent phase-space formulation of string theory [33-41] (and earlier, in the double field theory approach [42-46]). In this framework, it is not possible to derive a standard effective potential for some fields/moduli that produces vacuum expectation values for those fields. (Usually the vacuum is of a supersymmetric (AdS) kind and the de Sitter solution is a long lived excitation around that vacuum produced by some stringy configurations, such as brane-antibrane systems, and stabilized by fluxes [62-64]). In our proposal, we have, in principle, a cosmological de Sitter "blow-up" of a singular Minkowski background and a cosmologically induced supersymmetry breaking, and the hidden phase-space formulation operating behind the scenes and relating various energy scales via a natural see-saw like formula.

Viability. The 'axilaton' models (2.1) evade the oft-quoted no-go theorem [20, section 6] (see also ref. [21, pp. 480-482] or the recent exhaustive review [22, section 12.5]) primarily by being non-compact: following the stringy cosmic strings analysis [53, 54], we may compactify $\mathscr{Y}^{2}$ by including the limit-points $z \rightarrow \pm \infty$, and reexamine the behavior of the warp-factors over this now compact "internal" space. In particular, neither the $\Lambda_{b}>0$ warp

\footnotetext{
${ }^{11}$ The notion of S-folds was originally coined by Hull in [31] being the S-duality analog of the T-folds [29, $30,32]$.
} 
factors (2.4) nor their $\Lambda_{b}=0$ variant (2.5) vanish at $z \rightarrow \pm \infty$ as would be required of these singularities in a compactification of $\left(\mathscr{Y}^{2} \cup\{ \pm \infty\}\right) \approx \mathbb{P}^{1}[20$, section 6.2]. In addition, ref. [20, section 6.2] emphasizes the importance of higher-curvature terms in (1.1) - which are known to enable the evasion of the no-go theorem. It would be clearly desirable to determine their effect on the 'axilaton' deformation family of models, but this remains an open question for now. Similarly, we defer the precise F-theory/heterotic dual of these considerations, and expected evasion of the dual no-go results $[67,68]$ to a subsequent effort. ${ }^{12}$

In turn, this family of models are driven by a highly nontrivial source: the energymomentum tensor of the $\tau=\tau(\theta)$ configurations (2.2) is non-zero and forces the metric (2.1) to not be Ricci-flat (2.3). In particular, the axion-dilaton configuration (2.2) provides an exotic matter background, since its energy-momentum tensor is indefinite over $\mathscr{Y}^{2}$ [13]:

$$
\begin{aligned}
{\left[T_{\mu \nu}\right] } & =\left[T(r) \eta_{a b}\right] \oplus \operatorname{diag}\left[-\frac{1}{8} \omega^{2} r^{-2}, \frac{1}{8} \omega^{2} \ell^{-2}\right], \\
T(r) & =-\frac{1}{8} \omega^{2} \ell^{-2}\left[1+\frac{1}{8} \omega^{2} \log (r / \ell)\right](r / \ell)^{2+\frac{1}{8} \omega^{2}} \log (r / \ell)
\end{aligned}
$$

This violates several of the energy positivity conditions (but none within the brane-world $\mathscr{W}^{3,1}$ ), reminding of the standard characteristics of traversable Lorentzian wormholes [69].

\subsection{Stability}

Monodromy and discretuum. For the axion-dilaton configurations (2.2) to specify stringy rather than merely supergravity solutions, their parameters $b_{0}, \omega, g_{s}$ must be restricted so that $\tau=\tau(\theta)$ would exhibit an $\mathrm{SL}(2 ; \mathbb{Z})$-monodromy rather than a continuous $\mathrm{SL}(2 ; \mathbb{R})$-transformation. Thus furnishing discrete $\mathrm{SL}(2 ; \mathbb{Z})$-orbits and since $\operatorname{dim} \mathrm{SL}(2)=3$, the 3-parameter family of choices $(2.2) \tau=\tau\left(\theta ; b_{0}, \omega, g_{s}\right)$ is naturally expected to form a discretuum. Since the axion-dilaton system naturally couples to fluxes, the well-known eponymous string theory results [70-72] corroborate the discreteness of the 'axilaton' configurations (2.2), as do the general string theory expectations [73, 74]. While we are not aware of a rigorous proof, this is strongly supported by the results of refs. [75, 76]. This implies that most of the continuous $\left(b_{0}, \omega, g_{s}\right)$-parameter space is the non-stringy "swampland" - except for the discrete subset of $\mathrm{SL}(2 ; \mathbb{Z})$-isolated points within it.

With this in mind, the 'axilaton' family of models can vary continuously only via the metric parameters $z_{0}$ and $\ell$ (i.e., $\Lambda_{b}$ ), which are independent of $b_{0}, \omega, g_{s}$. Eqs. $(2.4),(2.6)$ and the $\mathscr{Y}^{2}$-geometry that they parametrize (see figure 1 ) and the fact that $z_{0}$ is at infinite proper distance [13] jointly imply that the transversal length-scale $\ell$ in $\mathscr{Y}^{2}$ remains the only continuously variable physically relevant parameter. As discussed above, in justifying (2.10), no definitive physical mechanism is known for stabilizing $\ell$, although there do exist arguments to this end both in field and in string theory [59-61]. This already provides the 'axilaton' models with an unexpected and high degree of stability.

\footnotetext{
${ }^{12}$ These standard no-go theorems follow within the context of supergravity and the stringy $\alpha^{\prime}$ corrections. We will give a general comment about how the non-commutative phase-space formulation of string theory goes around this standard set-up at the end of section 5 .
} 
Finally, compactifying Type IIB theory on some supersymmetry-preserving space $\mathscr{Y}^{4}$ does involve its various moduli, but the choice of $\mathscr{Y}^{4}$ and stabilization of its moduli is beyond our present scope. In turn, there is no modulus associated with the transverse space $\mathscr{Y}^{2}$ other than its volume-related size, $\ell$, to which we return shortly. The noncompact solutions for $\mathscr{Y}^{2}$ depicted in figure 1 are bi-holomorphic to either $\mathbb{C}^{*}$ (far left), a punctured disk with a boundary (middle) or to an annulus with boundaries (far right); each of those has an essentially unique complex structure and so can have no complex structure moduli. ${ }^{13}$ In fact, even the (re)compactified version of $\mathscr{Y}^{2} \rightarrow \mathbb{P}^{1}$ has no complex structure deformation, and is characterized only by its volume-related size, $\ell$.

RG-flow and dimensional transmutation. In fact, the above fairly geometric presentation of the construction implies more: it also indicates that the parameter $\ell$ is determined dynamically, by a variant of the well-known "dimensional transmutation" phenomenon. To this end, we reiterate that $\ell$ is a "free" parameter (turning up as an "integration constant" in solving a differential equation), and is necessary on dimensional grounds in the codimension-2 solution (2.1). It characterizes the behavior of the metric (2.1a), its curvature (2.3), and its value sets the volume-scale for the transverse plane $\mathscr{Y}^{2}$. Both qualitatively and computationally, this emergence of $\ell$ is analogous to the well-understood emergence of $\Lambda_{\mathrm{QCD}}$, which is determined by the Landau pole in the $\alpha_{\mathrm{QCD}}(q)$ coupling constant. Although the Landau pole of $\alpha_{\mathrm{QCD}}(q)$ is in momentum space, whereas the naked singularity (in the 'axilaton' configurations and so for the dilaton field) is located in positional space $\left(\mathscr{Y}^{2}\right)$, the principle and the mechanism are the same.

This analogy is indeed justified as it stems from the underlying fact that the $\mathscr{Y}^{2}$-metric (wherein $\ell$ locates the naked singularity) is determined by the Einstein equation - which is by Friedan's argument $[7,8]$ the (Gell-Mann-Low) $\beta$-function in the $1+1$-dimensional worldsheet QFT:

$$
\beta_{\mu \nu}(g / T)=-\frac{\epsilon}{T} g_{\mu \nu}+R_{\mu \nu}+\frac{1}{2} T R_{\mu \rho \sigma \kappa} R_{\nu}^{\rho \sigma \kappa}+O\left(T^{2}\right) .
$$

where the temperature $T$ is identified with $\alpha^{\prime}$ of string theory, and $\epsilon$ parametrizes dimensional regularization, and the metric and its curvature tensors explicitly depend on $\ell$; see (2.1a) and (2.3). This has been extended canonically to include other targetspacetime fields (dilaton, axion, and fermions) [77-80]; see also [81-83]. Their inclusion effectively modifies the $R_{\mu \nu}$-term in (3.2) so as to include the appropriate "Ricci-form" energy-momentum tensor $\widetilde{T}_{\mu \nu}$, which is for the 'axilaton' models given in (2.3) and explicitly includes $(\omega / 2 \ell)^{2}$.

Since $\omega$ is the discrete $\operatorname{SL}(2, \mathbb{Z})$-anisotropy of the 'axilaton' configuration (2.2), the numerical value of the only remaining parameter, $\ell$, must be determined dynamically, as a renormalization fixed point, and may only be estimated by perturbative computations. Thus, instead of seeking a potential for a modulus to be stabilized and to determine $\ell$ as a vev, akin to the Higgs mechanism, the above facts establish an analogue with the QCD route to the QFT topic of "hidden symmetry" and associated dynamical stabilization of $\ell$ :

\footnotetext{
${ }^{13}$ We should like to thank Paul Green for discussion on this matter.
} 
the concrete value of $\ell$ is determined by the vanishing of $\beta_{\mu \nu}(g / T)$, where higher- $T$ (i.e., $\alpha^{\prime}$ ) corrections correct any estimate.

Combined effect. On the geometric side, $\ell$ is determined (again, up to next order renormalization) simultaneously by both the area (volume) and the curvature of the transverse space $\mathscr{Y}^{2}$, taken from the explicit formula [15, eqs. (14)-(15)]:

$$
M_{D-2}^{D-4}=M_{D}^{D-2} 2 \pi \ell^{2}\left|z_{0}\right|^{-\frac{D-1}{2(D-2)}} \times \begin{cases}\Gamma\left(\frac{D-3}{2(D-2)} ; \frac{1}{z_{0}}\right) & \text { for } z_{0}>0 \\ \gamma\left(\frac{D-3}{2(D-2)} ; \frac{1}{z_{0}}\right) & \text { for } z_{0}<0\end{cases}
$$

where $\Gamma\left(\cdots ; \frac{1}{z_{0}}\right)$ and $\gamma\left(\cdots ; \frac{1}{z_{0}}\right)$ are the "big" and "little" complementary incomplete gammafunctions. Although the proper distance to the naked singularity is infinite and so $\ell$ independent, the proper area is finite and scales with $\ell^{2}$. In turn, $\ell$ also appears in the stressenergy-momentum tensor of the 'axilaton' system, and so at once characterizes both the (Ricci) curvature of $\mathscr{Y}^{2}$ and the ('axilaton') matter distribution that causes this curvature. Finally, note that the value of $\Lambda$ scales by the inverse of $\ell^{2}$, rather than the size of the $3+1$ dimensional brane-world. In particular, because $\ell$ is the fundamental scale introduced by dimensional transmutation in $z:=\log (r / \ell)$, as is familiar from the logarithmic behavior of the Green's function in codimension- 2 solutions, this allows for the inverse relation between the energy scale associated with $\Lambda$ and the scale associated with $G_{N}$. That is the underlying reason for the emergence of the see-saw relation (2.10) between the scale associated with $\Lambda$ and the $3+1$ dimensional Planck scale.

The above-outlined analogy between $\ell$ in position space and $\Lambda_{Q C D}$ in momentum space, as well as the dual determination of both the area of $\mathscr{Y}^{2}$ and its (Ricci) curvature by $\ell$ reflects the inherent S-duality of the 'axilaton' configuration, strong-dilaton coupling, and therefore a need to go beyond the standard and familiar EFT analysis. Such an approach has indeed been advanced recently, based on the inherently non-commutative phase-space approach [33-41] and relying on results in non-commutative QFT. This approach is naturally implemented in the case of our solution which is a deformation of a stringy cosmic string, and which can be therefore understood as an effective string theory (along the lines suggested by Polchinski and Strominger in [48]). The effective world-sheet description of our solution can be thus, to leading order, framed using the formalism of [33-41], which in turn leads to an effective non-commutative description of our solution in terms of doubled and generalized-geometric phase-space data. This then affords a complementary analysis, which does corroborate our present arguments for stability of $\ell$, but is both conceptually and technically beyond the present scope, and has been published separately [66].

\section{Novel features and consequences}

We now show that the anisotropy of (2.2) forces the 'axilaton' models to include the strong-coupling regime, and that these models also turn out to relate well-known (and supersymmetric) F-theory backgrounds [23, 24] and [25]. 


\subsection{Anisotropy}

The $\mathrm{SL}(2 ; \mathbb{Z})$ monodromy requires that $\left(g_{s}^{D} \stackrel{\text { def }}{=}\left\langle e^{-\Phi}\right\rangle_{\mathscr{W} \rtimes \mathscr{Y}^{2}}\right) \sim O(1)$, which also agrees with modular invariance: the 'axilaton' models require string theory to not be weakly coupled throughout $\mathscr{W} \rtimes \mathscr{Y}^{2}$. We therefore expect higher order corrections. Nevertheless, within the $(D-2)$-dimensional spacetime

$$
\text { @ } \mathscr{W}_{z=0}^{D-1,1}: \quad g_{s}^{D-2}=g_{s}^{D} \sqrt{\alpha^{\prime} / \operatorname{Vol}\left(\mathscr{Y}^{2}\right)} \ll 1,
$$

since $\operatorname{Vol}\left(\mathscr{Y}^{2}\right)$ has been shown to be large [12]: string theory is thus weakly coupled within $\mathscr{W}_{z=0}^{D-1,1}$, and therein the low-energy effective field theory approximation is well justified.

In going beyond the tree-level approximation consider evaluating the string theory scattering cross-section for any particular process in the $D$-dimensional spacetime, where $g_{s}^{D} \sim O(1)$. In this double expansion, ordered by powers of $\alpha^{\prime}$ and of $g_{s}$, the latter is equivalently ordered by the genus of the interacting worldsheet surface. Compare now the genus- $g$ contributions in any such computation with those at genus- $(g+1)$ - with everything else the same. In a straightforwardly pragmatic sense, the relative ratio of such two contributions provides a measure as to how reliable string-perturbative computations are, i.e., how weakly (or strongly) string theory is coupled; dub this the effective string coupling parameter $g_{s}^{\text {(eff) }}$.

All such ratios (for any particular physical process) will necessarily depend on the local value of the dilaton field ${ }^{14}$ (and possibly also the axion). In the 'axilaton' models, these fields vary over the $D$-dimensional spacetime, and so does then also this effective string coupling, $g_{s}^{\text {(eff) }}$. Notably, the 'axilaton' configurations (2.2) imply $g_{s}^{\text {(eff) }}<1$ in some $\theta$-directions in $\mathscr{Y}^{2}, g_{s}^{\text {(eff) }} \sim 1$ in others, and even $g_{s}^{\text {(eff) }}>1$ within the $\tau_{I}$ configuration:

$$
g_{s}^{\text {(eff) }}\left[\tau_{I}(\pi-\epsilon)\right] \ll 1 \quad \text { whereas } \quad g_{s}^{\text {(eff) }}\left[\tau_{I}(\pi+\epsilon)\right] \gg 1, \quad \text { for } \omega \sim O(1) .
$$

Because of the discontinuity of $\Delta \tau_{I}(\pi)=e^{2 \pi \omega}$, the 'axilaton' models thereby explicitly patch effectively weakly-coupled string theory to effectively (S-dual) strongly-coupled string theory across the $\theta=\pi$ direction in $\mathscr{Y}^{2} .{ }^{15}$ With $g_{s}^{D} \sim O(1)$ and by gluing regimes with reciprocally weak/strong effective string interactions (4.2), this S-duality patching makes it evident that the low-energy tree-level effective field theory approximation is sorely lacking.

Given the important role of modular invariance and the $\operatorname{SL}(2 ; \mathbb{Z})$ transformations for our solution, and in particular, the S-duality part of $\mathrm{SL}(2 ; \mathbb{Z})$, we want to draw an analogy with what is known about T-duality and T-folds in the context of the double field theory, and the phase-space formulation of string theory. We propose that our solution should be naturally viewed from a phase-space point of view, as an S-duality analog of the Tfold, that we call an S-fold, which glues the weakly and the strongly coupled regimes of our solution. ${ }^{16}$ In order to make sense of this picture, we need to include stringy degrees

\footnotetext{
${ }^{14}$ The "running" of coupling parameters, i.e., the dependence of the interaction strength on the colliding momenta is of course familiar in quantum field theory, and naturally translates also into a dual dependence on the collision proximity. While different in technical details, the fact that the dilaton and the string interaction strength can vary over the position (and momentum space) is conceptually the same.

${ }^{15}$ This is akin to the T-fold solutions [29-32], where local chart patching involves (T-duality) mirrorsymmetry.

${ }^{16}$ This use of S-folds has also recently occurred in the context of SCFT in various dimensions, e.g., [84]. It also resonates with the U-duality results in the so-called exceptional geometry [85-89].
} 
of freedom required for such a phase-space formulation that are not taken into account in the effective field theory discussion In particular, the usual identification of the target space being spanned by only the sum $\left\langle\hat{X}_{L}^{\mu}(\tau, \sigma)+\hat{X}_{R}^{\mu}(\tau, \sigma)\right\rangle$ should be amended. The Sdual (and strongly stringy-coupled) patching (4.2) indicates a need for (re)incorporating other stringy degrees of freedom, and at the very least also $\left\langle\hat{X}_{L}^{\mu}(\tau, \sigma)-\hat{X}_{R}^{\mu}(\tau, \sigma)\right\rangle$ : these vev's being determined by linear combinations of the canonically conjugate/dual Schrödinger center-of-mass operators [11] $\hat{x}^{\mu}$ and $\hat{p}^{\mu}\left(\tau / p^{+}\right)$implies the need to (re)incorporate the "momentum" space into this more complete description of the target space in string theory; we return to this in the next section. This then leads to a (non-commutative) phase-space geometry of the type discussed in [33-41].

\subsection{Holomorphic limits}

As indicated by the deformation from step 1 to 2 in the roadmap in section 2, the nonholomorphic axion-dilaton configurations (2.2) are a deformation of the holomorphic configuration achieved by removing the anisotropy: $\omega \rightarrow 0$. This results in the familiar and well understood type IIB orientifold limit of F-theory [23, 24] with $\tau=\alpha+i e^{-\Phi}=$ const.

However, starting with the configuration (2.2), it is also possible to find another novel holomorphic solution, by enforcing the Cauchy-Riemann conditions

$$
\frac{\partial u}{\partial r}=\frac{1}{r} \frac{\partial v}{\partial \theta} \quad \text { and } \quad \frac{\partial v}{\partial r}=-\frac{1}{r} \frac{\partial u}{\partial \theta}, \quad \text { for }\left\{\begin{array}{l}
u(r, \theta)=\Re[f(r, \theta)] \\
v(r, \theta)=\Im[f(r, \theta)]
\end{array}\right.
$$

in systematic iterations. For example and definiteness, start with $\tau_{I I}(\theta)=-\tau_{0} \tanh (\omega \theta)+$ $i \tau_{0} \operatorname{sech}(\omega \theta)$, i.e., with $u_{0}(r, \theta)=\tau_{0} \tanh (\omega \theta)$. The second of the Cauchy-Riemann conditions in (4.3) then implies that

$$
\begin{aligned}
\frac{\partial v}{\partial r} & =\frac{\tau_{0}}{r} \tanh ^{\prime}(\omega \theta), \quad \tanh ^{\prime}(\omega \theta) \stackrel{\text { def }}{=} \frac{\partial}{\partial \theta} \tanh (\omega \theta), \\
\text { so } \quad v_{1}(r, \theta) & =\tau_{0} \log (r) \tanh ^{\prime}(\omega \theta)+f(\theta),
\end{aligned}
$$

where $f(\theta)$ is an unknown integration $r$-constant. Solving in turn the first Cauchy-Riemann equation (4.3) for $u(r, \theta)$, we complete the first iteration:

$$
u_{1}(r, \theta)=\frac{1}{2} \tau_{0} \log ^{2}(r) \tanh ^{\prime \prime}(\omega \theta)+\log (r) f^{\prime}(\theta)+g(\theta),
$$

where $g(\theta)$ is another integration $r$-constant. This $u_{1}(r, \theta)$ matches the supersymmetric axion [25] in the limit $\omega \rightarrow 0$ upon choosing $\tau_{0} \mapsto n / 2 \pi \omega$ and $g(\theta) \mapsto u_{0}(r, \theta)=\tau_{0} \tanh (\omega \theta)$. With this $u_{1}(r, \theta)$, we compute $v_{2}(r, \theta)$ from the first and $u_{2}(r, \theta)$ from the second Cauchy Riemann equation (4.3), and so on. Expanding also in $\theta$, re-summing and combining with the result of the same procedure starting from $v_{0}(r, \theta)=\tau_{0} \operatorname{sech}(\omega \theta)$ produces:

$$
\tau_{I I}=\tau_{0}(\tanh (\omega \theta)+i \operatorname{sech}(\omega \theta)) \quad \rightarrow \quad \tau_{0}(\tanh [\omega(\theta-i z)]+i \operatorname{sech}[\omega(\theta-i z)])
$$


with $z=\log (r)$ (and $\ell=1$ for simplicity). The non-holomorphic configuration (2.2b) then has two distinct holomorphic limits, and relates them:

$$
\begin{aligned}
& {\left[b_{0}+\tau_{0}(\tanh (\omega \theta)+i \operatorname{sech}(\omega \theta))\right] \underset{\mid \omega \rightarrow \theta-i z}{\stackrel{\text { iterate }(4.3)}{\longrightarrow}}\left[b_{0}+\tau_{0}(\tanh [\omega(\theta-i z)]+i \operatorname{sech}[\omega(\theta-i z)])\right]} \\
& {\left[\left(\alpha, e^{-\Phi}\right)=\left(b_{0}, \tau_{0}\right)\right]_{[23,24]} \stackrel{\text { transition }}{\stackrel{(2.2 \mathrm{~b}): \tau_{I I}}{\longrightarrow}} \quad\left[\left(\left[b_{0}+\tau_{0} \omega \theta\right], \tau_{0}[1-\omega z]\right)\right]_{[25]} .}
\end{aligned}
$$

This then defines the 'axilaton' $\tau_{I I}$-transition, which relates the constant axion-dilaton configurations [23, 24] and the ("helicoidal axion") D7 instanton [25] after choosing $\tau_{0} \mapsto$ $\frac{n}{2 \pi} g_{s}^{-1}$ and identifying $\omega \mapsto g_{s}$. This also relates $(2.2 \mathrm{~b})$ to its "holomorphization" (4.7, top-right), implemented by the simple analytic continuation $\theta \rightarrow \theta-i \log (r / \ell)$, which then itself provides a (third) related holomorphic Ansatz for the axion-dilaton system, and so a candidate related supersymmetric configuration.

The same can be done with the other 'axilaton' solution (2.2a), also resulting in the straightforward analytic continuation $\tau_{I}(\theta) \rightarrow \tau_{I}(\theta-i z)$. The resulting analogous 'axilaton' $\tau_{I}$-transition is:

$$
\begin{aligned}
& \begin{array}{ccc}
{\left[b_{0}+i \tau_{0} e^{\omega \theta}\right.} & \stackrel{\text { iterate }(4.3)}{\downarrow_{\omega \rightarrow 0}} & {\left[\begin{array}{c}
b_{0}+i \tau_{0} e^{\omega(\theta-i z)} \\
\theta \rightarrow \theta-i z
\end{array}\right.} \\
\downarrow O(\omega)
\end{array} \\
& {\left[\left(\alpha, e^{-\Phi}\right)=\left(b_{0}, \tau_{0}\right)\right] \underset{(2.2 \mathrm{a}): \tau_{I}}{\stackrel{\text { transition }}{\longrightarrow}}\left(\left[b_{0}+\tau_{0} \omega z\right], \tau_{0}[1+\omega \theta]\right),}
\end{aligned}
$$

wherein the bottom-right corner, $O(\omega)$-configuration has a $\theta$ vs. $z \stackrel{\text { def }}{=} \log (r / \ell)$ dependance that is $(z, \theta) \rightarrow(-\theta, z)$ rotated from the one in $(4.7$, bottom-left).

\section{$5 \quad$ Generalizations and implications}

The above-discussed 'axilaton' models in fact have a natural generalization to other moduli, appear naturally within a generic Ricci-flat description of spacetime, and give further evidence to a possible dependence on additional stringy degrees of freedom.

Diversity. As indicated at the outset [12], analogous models can be built driven by other moduli fields, $\phi^{\alpha}$. This generalization then allows the $\mathscr{Y}^{4}$-moduli to (also) vary over $\mathscr{Y}^{2}=S^{1} \times \mathscr{Z}$. If $\phi^{\alpha}=\phi^{\alpha}(\theta)$ is again aperiodic and anisotropic, and since the WeilPetersson-Zamolodchikov metric in moduli spaces of Calabi-Yau varieties is the natural generalization of the Teichmüller metric [90], $\phi^{\alpha}(\theta)$ will have to exhibit a "mapping class group" monodromy, generalizing $\operatorname{SL}(2 ; \mathbb{Z})$; see, e.g., refs. [91, 92] for a concrete example. We then expect the dynamics and phenomenology to be similar to the one driven by $\tau=\tau(\theta)$. It of course remains to verify that $\ell \sim \operatorname{size}(\mathscr{Z})$ may be chosen so as to satisfy the experimental limits on extra dimensions while preserving other desirable phenomenological features.

For example, choosing $\phi=\phi(\theta)$ to be the size ("breathing") modulus of $\mathscr{Y}^{4}$, the model patches, akin to (4.2), the "large" and the "small" copies of $\mathscr{Y}^{4}$ across the identified endpoints of $\theta \in[-\pi, \pi]$. This is precisely the crux of the T-fold constructions [29-32]. Conversely then, it is natural to ask whether these already generalized constructions can 
be deformed akin to the 'axilaton' models. On the other hand, the corresponding analogue of the "holomorphization" (4.6), as implemented in (4.7) by the analytic continuation $\theta \rightarrow \theta-i \log (r / \ell)$ eerily reminds of the analytic continuation $J \rightarrow J+i B$ of the Kähler form, which has ever since [93-95] and especially [96, 97] become sine qua non, in the study of moduli spaces of Calabi-Yau $n$-folds.

Conversely then, the non-holomorphic and anisotropic configurations (2.2) driving the 'axilaton' deformation family of models $(2.1)+(2.4)$ are easily seen to be analogous to the (exceptional) $B \rightarrow 0$ limit in the by now much better understood moduli space of Calabi-Yau $n$-folds. The fact that it is this non-holomorphic and anisotropic configuration (2.2) that also parametrizes both supersymmetry breaking and the possibility of the Minkowski $\rightarrow$ de Sitter deformation warrants a closer analysis of these 'axilaton' models.

Genericity. The Euclidean analytic continuation of the 9+1-dimensional spacetime in the type IIB string theory has to be Ricci-flat (and complex owing to its $N=2$ supersymmetry), whether compact or not, and hence is a (possibly non-compact) complex 5-dimensional product of Calabi-Yau and abelian factors. This certainly resonates with the geometric quantization conclusion, that Ricci-flatness, complex and Kähler structures in the loop-space all appear in "the string equation of motion" [98-103].

Just as Calabi-Yau 3-folds generically contain many isolated $\mathbb{P}^{1}$ 's, the so-called $\mathcal{O}(-1,-1)$-curves, which are small resolutions of nodes, (i.e., double-points, $A_{1}$ singularities, conifold singularities) [104], Calabi-Yau 5-folds generically contain many isolated Fano $\left(c_{1}>0\right)$ compact complex surfaces $\mathcal{S}$ [47]. For a Calabi-Yau 3-fold, these exceptional $\mathcal{O}(-1,-1)$-curves have a bulk Kähler metric which is generally null, but is straightforwardly deformed into a positive metric by adding a multiple of the intrinsic volume form [58]. Analogously, the bulk Kähler metric of the above Calabi-Yau 5-fold is null on the exceptional complex surfaces $\mathcal{S}$, but is straightforwardly deformed into a positive metric by adding a multiple of the Kähler metric specified by the intrinsic volume form of $\mathcal{S}$, which we dub the "bulk+local metric deformation."

Analytically continuing this Euclideanized Ricci-flat 10-fold back to a 9+1-dimensional spacetime, at least some of the generically occurring exceptional complex surfaces $\mathcal{S}$ within Calabi-Yau 5-fold will map to 3+1-dimensional sub-spacetimes. Within these 3+1-dimensional spacetime bubbles, the "bulk+local metric deformation" would naturally correspond to the Minkowski $\rightarrow$ de Sitter desingularization deformation discussed in section 2, with $\Lambda_{b}>0$ parametrizing the size of the analytically continued $\mathcal{S}$. This is indeed the deformation employed within the uncompactified four dimensional spacetime $\mathscr{W}^{3,1}$ in section 2 , as discussed right after (2.5). We conjecture that a very similar, "de Sitter-izing," supersymmetry breaking metric deformation may be employed within at least some of the generically plentiful exceptional surfaces $\mathcal{S}$.

\subsection{Seeing double}

In any worldsheet field theory underlying superstring theory, the large modes (with wavelengths $\lambda>\ell_{s}:=\sqrt{\alpha^{\prime}}$ ) of both coordinate fields $\hat{X}_{L}^{\mu}, \hat{X}_{R}^{\mu}$ probe the target space $\mathscr{X}$, whereas stringy-small modes (with $\lambda<\ell_{s}:=\sqrt{\alpha^{\prime}}$ ) of both $\hat{X}_{L}^{\mu}, \hat{X}_{R}^{\mu}$ probe $\widetilde{\mathscr{X}}$, the mirror spacetime. 


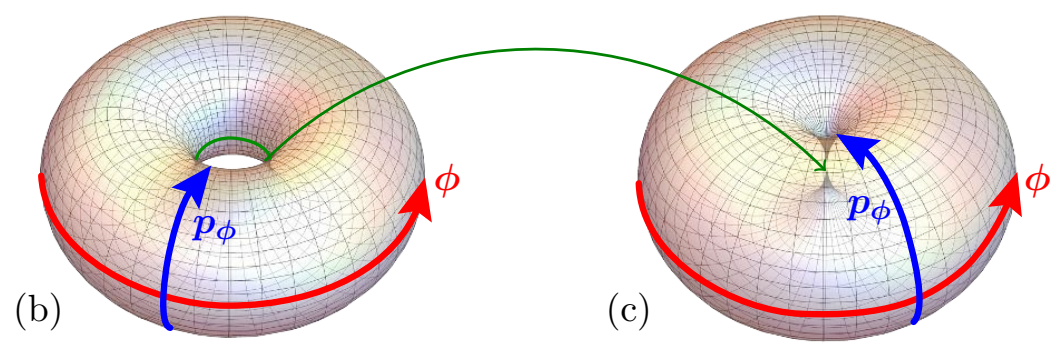

Figure 2. The phase-space of a point particle moving along a circle with fixed radius, at finite speed in b), and at infinite speed in c).

The full target space of string theory is therefore (locally) a product of these two factors, the latter of which is naturally identified to be the (T-dual) mirror of the former. This strongly resonates with the phase-space theory discussed at the end of section 4 and in [33-41], which may be justified conceptually also as follows:

1. Consider a point-particle moving on a rigid circle.

(a) At first blush, the phase-space of a point-particle on a circle is a cylinder, where the (vertical) $\mathbb{R}^{1}$-like $p_{\phi}$-generator represents momentum, at each point of the circle of possible positions, $\phi$.

(b) However, $p_{\phi} \rightarrow+\infty$ and $p_{\phi} \rightarrow-\infty$ are indistinguishable: if one moves infinitely fast, it does not matter in which direction one is moving. This compactifies the momentum direction into a circle, and the phase-space into a ring-torus (adding a copy of the position-circle at $p_{\phi}$-infinity).

(c) However, when one moves infinitely fast, one is simultaneously everywhere, so that the positional circle at $p_{\phi}$-infinity shrinks to a point.

The final, (b) $\rightarrow$ (c) step in this progression of modeling the phase-space of a pointparticle on a circle thus looks as in figure 2 .

2. The configuration space of a particle moving on an $n$-torus is $T^{n}=\mathbb{R}^{n} / \Lambda$, whereas its momentum space is naturally the (mirror) dual torus, $\widetilde{T}^{n}=\mathbb{R}^{n} / \Lambda^{*}$. This makes the phase-space into $T^{2 n} \sim \mathbb{R}^{2 n} /\left(\Lambda \oplus \Lambda^{*}\right)$, - except that again the total space is singular "at infinity."

3. For Calabi-Yau varieties, there exists a conjecture: ${ }^{17}$

(a) a completion of each fiber of $T_{M}^{*}$ is a mirror of the Calabi-Yau variety $M$;

(b) a completion of $T_{M}^{*}$ is a "double Calabi-Yau space," singular "at infinity."

This would imply the "true home" for the above-cited phase-space $(x, \tilde{x})$-geometry to be such a singular foliation, $\mathscr{X} * \widetilde{\mathscr{X}}$, of the usual spacetime $\mathscr{X}$ and its mirror $\widetilde{\mathscr{X}}$ : locally at any generic point, $\mathscr{X} * \widetilde{\mathscr{X}}$ looks like a product, but over certain locations in one factor, the other may singularize. By mirror symmetry, neither of the two factors is a preferred base of a fibration, and the total space may well be singular — as is the right-hand side illustration in figure 2 . Furthermore, $\mathscr{X} * \widetilde{\mathscr{X}}$ is naturally endowed with a non-commutativity structure induced ultimately from the symplectic position-momentum structure on the worldsheet,

\footnotetext{
${ }^{17}$ Dec. 1993, Lexington: http://www.ams.org/meetings/sectional/1890_program_ssh.html\#title.
} 
as employed in [33-41]. Incidentally, this target space doubling is also suggested from a closer look at geometric quantization, since it in fact involves the oriented (and so doubled) loop-space [98-103]. This seems to resonate with the considerable work in the last decade on the relation between non-commutativity and T-folds, S-folds, and "non- geometry" in general; see [105-108] and references therein. Separately, Calabi-Yau manifolds have been shown to independently appear in the momentum space of physical processes; see [109, 110] and references therein. Clearly, the $\mathbb{R}^{3,1}$-factor and its dual in $\mathscr{X} * \widetilde{\mathscr{X}}$ are diffeomorphic, but may well have different - and presumably complementary - metric properties, the study of which we defer for now.

\subsection{More on stability}

To recap, our 'axilaton' models that realize a positive cosmological constant within string theory depend on two types of parameters: the $b_{0}, \omega, g_{s}$ parametrizing the axion-dilaton system (2.2) are restricted by modular invariance to a discrete subset of $\mathrm{SL}(2 ; \mathbb{Z})$-isolated points. In turn, the metric (2.1)-(2.4) depends on $\ell$, which we argued can be stabilized dynamically by dimensional transmutation due to the codimension- 2 nature of the models at hand.

Second, the see-saw formula (2.10) for the cosmological constant in our model can be also understood as an example of a formula required by T-duality, given, first, the scale of non-commutativity of the phase-space formulation [33-41] (set by an effective size of the string), and captured by the parameter $\omega$ in our model, and, second, given the Planck scale (set by the value of the dilaton, viewed as the relevant volume form of the stringy phase-space), and captured by the parameter $\ell$ of our model.

More intuitively, the stringy de Sitter space can be understood as a blow-up in the "Calabi-Yau-ization" of the 10 dimensional Minkowski habitat of string theory, as implied by the "Einstein equation" in the stringy loop space, which implies an infinite dimensional Ricci-flat/Calabi-Yau nature of the stringy configuration space [98-103]. The value of the cosmological constant is then the size of the relevant blow-up fixed by the requirements of T-duality ("mirror symmetry") in the phase-space reformulation of the geometry of the stringy loop space. Then the basic idea is that the stringy stability of a "stringy de Sitter spacetime" follows from an optimization between the short- and the long-distance spacetime physics emerging from the phase-space formulation of string theory, the geometry of which is really responsible for the appearance of a positive cosmological constant in the first place.

The actual construction reviewed in this paper can be therefore understood as an illustrative toy model for this new, intrinsically non-commutative phase-space picture of string theory that naturally leads to de Sitter backgrounds, and which we hope to explore in more detail in a sequel to this note.

\section{Summary, outlook and conclusions}

In this paper, we argue that the 'axilaton' models [12-17] imply a dependence on additional stringy degrees of freedom, and are related to the recently uncovered non-commutatively 
doubled phase-space formulation of string theory [33-41]. Furthermore, this improves the understanding of how de Sitter spacetimes may be realized in string theory.

In particular, (1) the driving sources (2.2) admit a "holomorphization" (4.6), and thereby two separate supersymmetric limits (4.7), whereby the 'axilaton' configurations (2.2) relate these two distinct and well known supersymmetric configurations, as well as the third holomorphic and possibly supersymmetric configuration (4.6). In addition, (2) the aperiodic anisotropy of the axion-dilaton configuration (2.2) exhibits a type of chart-patching (4.2) that explicitly employs S-duality, and so implies that the 'axilaton' models cannot be limited to weak string coupling. It is important to note that (3) the 'axilaton' configurations (2.2) may equally well be used for other moduli, where the aperiodic anisotropy implies chart-patching (4.2) that employs T-duality — strikingly similar to the T-folds [29-32]. Also, (4) the "holomorphization" (4.6) bears a strikingly similarity to the by now very well understood $J \rightarrow J+i B$ analytic continuation of Kähler moduli spaces of Calabi-Yau $n$-folds. By converse, the physics driven by the 'axilaton' configurations are then fairly ubiquitous, and correspond to the $B \rightarrow 0$ limit in the moduli space of Calabi-Yau $n$-folds. Furthermore, (5) the Euclidean version of the Minkowski $\rightarrow$ de Sitter deformation within the $3+1$ dimensional spacetime $\mathscr{W}_{z=0}^{3,1}$ seems strikingly similar to the deformation of the Kähler metric in the blowup or small resolution exceptional sets. Since exceptional complex 2-folds are ubiquitous (as small resolutions of nodes) within CalabiYau (complex) 5-folds, in a converse Lorentzian analytic continuation, at least some of those complex 2-folds could serve as 3+1-dimensional (sub)spacetimes - and should admit a de Sitter metric à la $(2.1)+(2.4)$. Finally, (6) the value of $\Lambda(2.7)$ scales as $\ell^{-2}$, where $\ell$ is the fundamental scale introduced by dimensional transmutation in $z:=\log (r / \ell)$ in (2.1). That is the underlying reason for the emergence of the see-saw relation between the scale associated with $\Lambda$ and the $3+1$ dimensional Planck scale.

As explained in the main body of the paper, the 'axilaton' solutions discussed herein can be viewed as a well-defined deformation of the stringy cosmic strings/branes in type IIB/F-theory, and the latter can, at least in principle, be related to a deconstruction of the cosmological constant from 3- to 4-dimensional spacetime. What is meant here is the old observation of Witten [111] about the peculiar features of supersymmetry in 3 -dimensional spacetime, where due to the presence of conical defects in 3-dimensional gravity, the supercharges do not have to be globally defined, so one has supersymmetry but not the degeneracy in masses between bosons and fermions - the mass splitting is controlled by the strength of the conical defect. By deconstruction (performed in [112], but also discussed in [113]), one can obtain a 4-dimensional version of Witten's argument, albeit now with stringy defects. These stringy defects are generically strongly coupled in the 4dimensional continuum limit, and could be naturally related to the non-supersymmetric stringy cosmic strings. So, in principle, our construction can be connected to this narrative, even though the details remain to be worked out.

Finally, as discussed at the end of section 5 and motivating a sequel to this note [66], the latter few of the above observations imply that the familiar point-field limit description of spacetime is in fact incomplete: more of the stringy degrees of freedom must be included, extending the stringy target space into a certain double, tentatively modeled on the corresponding phase-space. 


\section{Acknowledgments}

We thank Lara Anderson and James Gray for interesting comments on string compactifications. DM thanks Laurent Freidel and Rob Leigh for numerous insightful discussions over many years on the topic of quantum gravity and string theory, and Vijay Balasubramanian, Petr Hořava and Jan de Boer for many conversations on de Sitter spacetime and string theory. We should also like to thank the anonymous Referee for constructive criticism. PB would like to thank the CERN Theory Group for their hospitality over the past several years. TH is grateful to the Department of Physics, University of Maryland, College Park MD, and the Physics Department of the Faculty of Natural Sciences of the University of Novi Sad, Serbia, for the recurring hospitality and resources. The work of DM is supported in part by the Julian Schwinger Foundation.

Open Access. This article is distributed under the terms of the Creative Commons Attribution License (CC-BY 4.0), which permits any use, distribution and reproduction in any medium, provided the original author(s) and source are credited.

\section{References}

[1] Supernova Search Team collaboration, Observational evidence from supernovae for an accelerating universe and a cosmological constant, Astron. J. 116 (1998) 1009 [astro-ph/9805201] [INSPIRE].

[2] Supernova Cosmology Project collaboration, Measurements of $\Omega$ and $\Lambda$ from 42 high redshift supernovae, Astrophys. J. 517 (1999) 565 [astro-ph/9812133] [INSPIRE].

[3] U.H. Danielsson and T. Van Riet, What if string theory has no de Sitter vacua?, Int. J. Mod. Phys. D 27 (2018) 1830007 [arXiv:1804.01120] [inSPIRE].

[4] M. Cicoli, S. De Alwis, A. Maharana, F. Muia and F. Quevedo, De Sitter vs quintessence in string theory, Fortsch. Phys. 67 (2019) 1800079 [arXiv: 1808.08967] [INSPIRE].

[5] G. Obied, H. Ooguri, L. Spodyneiko and C. Vafa, De Sitter space and the swampland, arXiv: 1806.08362 [INSPIRE].

[6] P. Agrawal, G. Obied, P.J. Steinhardt and C. Vafa, On the cosmological implications of the string swampland, Phys. Lett. B $\mathbf{7 8 4}$ (2018) 271 [arXiv: 1806.09718] [INSPIRE].

[7] D.H. Friedan, Nonlinear models in two $+\epsilon$ dimensions, Annals Phys. 163 (1985) 318 [INSPIRE].

[8] D. Friedan, Nonlinear models in two $\epsilon$ dimensions, Phys. Rev. Lett. 45 (1980) 1057 [INSPIRE].

[9] B. Zwiebach, A first course in string theory, Cambridge University Press, Cambridge, U.K. (2004) [INSPIRE].

[10] M.B. Green, J.H. Schwarz and E. Witten, Superstring theory. Volume 1: introduction, Cambridge Monographs on Mathematical Physics, Cambridge University Press, Cambridge, U.K. (1987) [INSPIRE].

[11] J. Polchinski, String theory, Cambridge Monographs on Mathematical Physics, Cambridge University Press, Cambridge, U.K. (1998). 
[12] P. Berglund, T. Hübsch and D. Minić, Exponential hierarchy from space-time variable string vacua, JHEP 09 (2000) 015 [hep-th/0005162] [INSPIRE].

[13] P. Berglund, T. Hübsch and D. Minić, Probing naked singularities in nonsupersymmetric string vacua, JHEP 02 (2001) 010 [hep-th/0012042] [INSPIRE].

[14] P. Berglund, T. Hübsch and D. Minić, On relativistic brane probes in singular space-times, JHEP 01 (2001) 041 [hep-th/0012180] [INSPIRE].

[15] P. Berglund, T. Hübsch and D. Minić, Localized gravity and large hierarchy from string theory?, Phys. Lett. B 512 (2001) 155 [hep-th/0104057] [INSPIRE].

[16] P. Berglund, T. Hübsch and D. Minić, De Sitter space-times from warped compactifications of IIB string theory, Phys. Lett. B 534 (2002) 147 [hep-th/0112079] [INSPIRE].

[17] P. Berglund, T. Hübsch and D. Minić, Relating the cosmological constant and supersymmetry breaking in warped compactifications of IIB string theory, Phys. Rev. D 67 (2003) 041901 [hep-th/0201187] [INSPIRE].

[18] G.W. Gibbons and C.M. Hull, De Sitter space from warped supergravity solutions, hep-th/0111072 [INSPIRE].

[19] G.W. Gibbons, Aspects of supergravity theories, in XV GIFT seminar on supersymmetry and supergravity, Gerona, Spain, 4-11 June 1984 [INSPIRE].

[20] J.M. Maldacena and C. Núñez, Supergravity description of field theories on curved manifolds and a no go theorem, Int. J. Mod. Phys. A 16 (2001) 822 [hep-th/0007018] [INSPIRE].

[21] K. Becker, M. Becker and J.H. Schwarz, String theory and M-theory: a modern introduction, Cambridge University Press, Cambridge, U.K. (2007) [INSPIRE].

[22] U. Gran, J. Gutowski and G. Papadopoulos, Classification, geometry and applications of supersymmetric backgrounds, Phys. Rept. 794 (2019) 1 [arXiv:1808.07879] [INSPIRE].

[23] C. Vafa, Evidence for F-theory, Nucl. Phys. B 469 (1996) 403 [hep-th/9602022] [InSPIRE].

[24] A. Sen, F-theory and orientifolds, Nucl. Phys. B 475 (1996) 562 [hep-th/9605150] [INSPIRE].

[25] M.B. Einhorn and L.A. Pando Zayas, On seven-brane and instanton solutions of type IIB, Nucl. Phys. B 582 (2000) 216 [hep-th/0003072] [INSPIRE].

[26] D. Andriot, On the de Sitter swampland criterion, Phys. Lett. B 785 (2018) 570 [arXiv: 1806.10999] [INSPIRE].

[27] J.J. Heckman, C. Lawrie, L. Lin and G. Zoccarato, F-theory and dark energy, Fortsch. Phys. 67 (2019) 1900057 [arXiv: 1811.01959] [INSPIRE].

[28] J.J. Heckman, C. Lawrie, L. Lin, J. Sakstein and G. Zoccarato, Pixelated dark energy, Fortsch. Phys. 67 (2019) 1900071 [arXiv:1901.10489] [INSPIRE].

[29] A. Dabholkar and C. Hull, Duality twists, orbifolds and fluxes, JHEP 09 (2003) 054 [hep-th/0210209] [INSPIRE].

[30] A. Flournoy, B. Wecht and B. Williams, Constructing nongeometric vacua in string theory, Nucl. Phys. B 706 (2005) 127 [hep-th/0404217] [INSPIRE].

[31] C.M. Hull, A geometry for non-geometric string backgrounds, JHEP 10 (2005) 065 [hep-th/0406102] [INSPIRE]. 
[32] C. Hull, D. Israel and A. Sarti, Non-geometric Calabi-Yau backgrounds and K3 automorphisms, JHEP 11 (2017) 084 [arXiv:1710.00853] [INSPIRE].

[33] L. Freidel, R.G. Leigh and D. Minić, Born reciprocity in string theory and the nature of spacetime, Phys. Lett. B 730 (2014) 302 [arXiv:1307.7080] [INSPIRE].

[34] L. Freidel, R.G. Leigh and D. Minić, Quantum gravity, dynamical phase space and string theory, Int. J. Mod. Phys. D 23 (2014) 1442006 [arXiv:1405.3949] [inSPIRE].

[35] L. Freidel, R.G. Leigh and D. Minić, Metastring theory and modular space-time, JHEP 06 (2015) 006 [arXiv: 1502.08005] [INSPIRE].

[36] L. Freidel, R.G. Leigh and D. Minić, Modular spacetime, Int. J. Mod. Phys. D 24 (2015) 1544028 [INSPIRE].

[37] L. Freidel, R.G. Leigh and D. Minić, Quantum spaces are modular, Phys. Rev. D 94 (2016) 104052 [arXiv: 1606.01829] [INSPIRE].

[38] L. Freidel, R.G. Leigh and D. Minić, Modular spacetime and metastring theory, J. Phys. Conf. Ser. 804 (2017) 012032 [INSPIRE].

[39] L. Freidel, R.G. Leigh and D. Minić, Intrinsic non-commutativity of closed string theory, JHEP 09 (2017) 060 [arXiv:1706.03305] [INSPIRE].

[40] L. Freidel, R.G. Leigh and D. Minić, Noncommutativity of closed string zero modes, Phys. Rev. D 96 (2017) 066003 [arXiv: 1707.00312] [INSPIRE].

[41] L. Freidel, J. Kowalski-Glikman, R.G. Leigh and D. Minić, Theory of metaparticles, Phys. Rev. D 99 (2019) 066011 [arXiv: 1812.10821] [INSPIRE].

[42] A.A. Tseytlin, Duality symmetric formulation of string world sheet dynamics, Phys. Lett. B 242 (1990) 163 [INSPIRE].

[43] A.A. Tseytlin, Duality symmetric closed string theory and interacting chiral scalars, Nucl. Phys. B 350 (1991) 395 [inSPIRE].

[44] W. Siegel, Superspace duality in low-energy superstrings, Phys. Rev. D 48 (1993) 2826 [hep-th/9305073] [INSPIRE].

[45] W. Siegel, Two vierbein formalism for string inspired axionic gravity, Phys. Rev. D 47 (1993) 5453 [hep-th/9302036] [INSPIRE].

[46] C. Hull and B. Zwiebach, Double field theory, JHEP 09 (2009) 099 [arXiv:0904.4664] [INSPIRE].

[47] T. Hübsch, A hitchhiker's guide to superstring jump gates and other worlds, Nucl. Phys. Proc. Suppl. A 52 (1997) 347 [INSPIRE].

[48] J. Polchinski and A. Strominger, Effective string theory, Phys. Rev. Lett. 67 (1991) 1681 [INSPIRE].

[49] E. Silverstein, (A)dS backgrounds from asymmetric orientifolds, Clay Mat. Proc. 1 (2002) 179 [hep-th/0106209] [INSPIRE].

[50] A. Maloney, E. Silverstein and A. Strominger, De Sitter space in noncritical string theory, in The future of theoretical physics and cosmology: celebrating Stephen Hawking's $60^{\text {th }}$ birthday. Proceedings, Workshop and Symposium, Cambridge, U.K., 7-10 January 2002, pg. 570 [hep-th/0205316] [INSPIRE]. 
[51] G. 't Hooft, On the phase transition towards permanent quark confinement, Nucl. Phys. B 138 (1978) 1 [INSPIRE].

[52] G. 't Hooft, A property of electric and magnetic flux in non-Abelian gauge theories, Nucl. Phys. B 153 (1979) 141 [INSPIRE].

[53] B.R. Greene, A.D. Shapere, C. Vafa and S.-T. Yau, Stringy cosmic strings and noncompact Calabi-Yau manifolds, Nucl. Phys. B 337 (1990) 1 [inSPIRE].

[54] P.S. Green and T. Hübsch, Space-time variable superstring vacua (Calabi-Yau cosmic yarn), Int. J. Mod. Phys. A 9 (1994) 3203 [hep-th/9306057] [inSPIRE].

[55] R. Gregory, Nonsingular global strings, Phys. Rev. D 54 (1996) 4955 [gr-qc/9606002] [INSPIRE].

[56] R. Gregory, Nonsingular global string compactifications, Phys. Rev. Lett. 84 (2000) 2564 [hep-th/9911015] [INSPIRE].

[57] N. Kaloper, Bent domain walls as brane worlds, Phys. Rev. D 60 (1999) 123506 [hep-th/9905210] [INSPIRE].

[58] P. Green and T. Hübsch, Calabi-Yau hypersurfaces in products of semiample surfaces, Commun. Math. Phys. 115 (1988) 231 [INSPIRE].

[59] I. Antoniadis, R. Minasian and P. Vanhove, Noncompact Calabi-Yau manifolds and localized gravity, Nucl. Phys. B 648 (2003) 69 [hep-th/0209030] [INSPIRE].

[60] G.R. Dvali and G. Gabadadze, Gravity on a brane in infinite volume extra space, Phys. Rev. D 63 (2001) 065007 [hep-th/0008054] [INSPIRE].

[61] E. Kiritsis, N. Tetradis and T.N. Tomaras, Induced brane gravity: realizations and limitations, JHEP 08 (2001) 012 [hep-th/0106050] [INSPIRE].

[62] S. Kachru, R. Kallosh, A.D. Linde and S.P. Trivedi, De Sitter vacua in string theory, Phys. Rev. D 68 (2003) 046005 [hep-th/0301240] [INSPIRE].

[63] V. Balasubramanian, P. Berglund, J.P. Conlon and F. Quevedo, Systematics of moduli stabilisation in Calabi-Yau flux compactifications, JHEP 03 (2005) 007 [hep-th/0502058] [INSPIRE].

[64] M.R. Douglas and S. Kachru, Flux compactification, Rev. Mod. Phys. 79 (2007) 733 [hep-th/0610102] [INSPIRE].

[65] N. Kaloper and A. Padilla, Vacuum energy sequestering: the framework and its cosmological consequences, Phys. Rev. D 90 (2014) 084023 [Addendum ibid. D 90 (2014) 109901] [arXiv: 1406.0711] [INSPIRE].

[66] P. Berglund, T. Hübsch and D. Minić, Dark energy and string theory, Phys. Lett. B 798 (2019) 134950 [arXiv: 1905.08269] [INSPIRE].

[67] S.R. Green, E.J. Martinec, C. Quigley and S. Sethi, Constraints on string cosmology, Class. Quant. Grav. 29 (2012) 075006 [arXiv:1110.0545] [InSPIRE].

[68] D. Kutasov, T. Maxfield, I. Melnikov and S. Sethi, Constraining de Sitter space in string theory, Phys. Rev. Lett. 115 (2015) 071305 [arXiv:1504.00056] [INSPIRE].

[69] M. Visser, Lorentzian wormholes: from Einstein to Hawking, AIP Series in Computational and Applied Mathematical Physics, American Institute of Physics, U.S.A. (1996). 
[70] J. Polchinski and M.J. Strassler, The string dual of a confining four-dimensional gauge theory, hep-th/0003136 [INSPIRE].

[71] R. Bousso and J. Polchinski, Quantization of four form fluxes and dynamical neutralization of the cosmological constant, JHEP 06 (2000) 006 [hep-th/0004134] [INSPIRE].

[72] S.B. Giddings, S. Kachru and J. Polchinski, Hierarchies from fluxes in string compactifications, Phys. Rev. D 66 (2002) 106006 [hep-th/0105097] [INSPIRE].

[73] J. Polchinski, Monopoles, duality and string theory, Int. J. Mod. Phys. A 19S1 (2004) 145 [hep-th/0304042] [INSPIRE].

[74] T. Banks and N. Seiberg, Symmetries and strings in field theory and gravity, Phys. Rev. D 83 (2011) 084019 [arXiv: 1011.5120] [INSPIRE].

[75] D. Harlow and H. Ooguri, Symmetries in quantum field theory and quantum gravity, arXiv: 1810.05338 [INSPIRE].

[76] D. Harlow and H. Ooguri, Constraints on symmetries from holography, Phys. Rev. Lett. 122 (2019) 191601 [arXiv: 1810.05337] [INSPIRE].

[77] E.S. Fradkin and A.A. Tseytlin, Effective field theory from quantized strings, Phys. Lett. B 158 (1985) 316 [INSPIRE].

[78] E.S. Fradkin and A.A. Tseytlin, Quantum string theory effective action, Nucl. Phys. B 261 (1985) 1 [Erratum ibid. B 269 (1986) 745] [INSPIRE].

[79] A. Sen, Equations of motion for the heterotic string theory from the conformal invariance of the $\sigma$-model, Phys. Rev. Lett. 55 (1985) 1846 [INSPIRE].

[80] C.G. Callan Jr., E.J. Martinec, M.J. Perry and D. Friedan, Strings in background fields, Nucl. Phys. B 262 (1985) 593 [inSPIRE].

[81] L. Álvarez-Gaumé and D.Z. Freedman, Geometrical structure and ultraviolet finiteness in the supersymmetric $\sigma$-model, Commun. Math. Phys. 80 (1981) 443 [InSPIRE].

[82] L. Álvarez-Gaumé, D.Z. Freedman and S. Mukhi, The background field method and the ultraviolet structure of the supersymmetric nonlinear $\sigma$-model, Annals Phys. 134 (1981) 85 [INSPIRE].

[83] S. Mukhi, The geometric background field method, renormalization and the Wess-Zumino term in nonlinear $\sigma$-models, Nucl. Phys. B 264 (1986) 640 [INSPIRE].

[84] O. Aharony and Y. Tachikawa, S-folds and $4 d N=3$ superconformal field theories, JHEP 06 (2016) 044 [arXiv: 1602.08638] [INSPIRE].

[85] M. Cederwall, J. Edlund and A. Karlsson, Exceptional geometry and tensor fields, JHEP 07 (2013) 028 [arXiv: 1302.6736] [inSPIRE].

[86] O. Hohm and H. Samtleben, Exceptional form of D=11 supergravity, Phys. Rev. Lett. 111 (2013) 231601 [arXiv:1308.1673] [INSPIRE].

[87] O. Hohm and H. Samtleben, Exceptional field theory I: $E_{6(6)}$ covariant form of M-theory and type IIB, Phys. Rev. D 89 (2014) 066016 [arXiv:1312.0614] [INSPIRE].

[88] O. Hohm and H. Samtleben, Exceptional field theory II: $E_{7(7)}$, Phys. Rev. D 89 (2014) 066017 [arXiv: 1312.4542] [INSPIRE].

[89] O. Hohm and H. Samtleben, Exceptional field theory III: $E_{8(8)}$, Phys. Rev. D 90 (2014) 066002 [arXiv: 1406.3348] [INSPIRE]. 
[90] P. Candelas, T. Hübsch and R. Schimmrigk, Relation between the Weil-Petersson and Zamolodchikov metrics, Nucl. Phys. B 329 (1990) 583 [inSPIRE].

[91] P. Candelas, X.C. De La Ossa, P.S. Green and L. Parkes, A pair of Calabi-Yau manifolds as an exactly soluble superconformal theory, Nucl. Phys. B 359 (1991) 21 [InSPIRE].

[92] P. Candelas, X.C. De la Ossa, P.S. Green and L. Parkes, An exactly soluble superconformal theory from a mirror pair of Calabi-Yau manifolds, Phys. Lett. B 258 (1991) 118 [INSPIRE].

[93] P.S. Aspinwall, B.R. Greene and D.R. Morrison, Space-time topology change and stringy geometry, J. Math. Phys. 35 (1994) 5321 [inSPIRE].

[94] P.S. Aspinwall, B.R. Greene and D.R. Morrison, Measuring small distances in $N=2$ $\sigma$-models, Nucl. Phys. B 420 (1994) 184 [hep-th/9311042] [INSPIRE].

[95] P.S. Aspinwall, B.R. Greene and D.R. Morrison, Calabi-Yau moduli space, mirror manifolds and space-time topology change in string theory, Nucl. Phys. B 416 (1994) 414 [hep-th/9309097] [INSPIRE].

[96] E. Witten, Phases of $N=2$ theories in two-dimensions, Nucl. Phys. B 403 (1993) 159 [hep-th/9301042] [INSPIRE].

[97] D.R. Morrison and M.R. Plesser, Summing the instantons: quantum cohomology and mirror symmetry in toric varieties, Nucl. Phys. B 440 (1995) 279 [hep-th/9412236] [INSPIRE].

[98] I.B. Frenkel, H. Garland and G.J. Zuckerman, Semiinfinite cohomology and string theory, Proc. Nat. Acad. Sci. 83 (1986) 8442 [inSPIRE].

[99] M.J. Bowick and S.G. Rajeev, String theory as the Kähler geometry of loop space, Phys. Rev. Lett. 58 (1987) 535 [Erratum ibid. 58 (1987) 1158] [INSPIRE].

[100] M.J. Bowick and S.G. Rajeev, The holomorphic geometry of closed bosonic string theory and Diff $S^{1} / S^{1}$, Nucl. Phys. B 293 (1987) 348 [InSPIRE].

[101] M.J. Bowick and S. Rajeev, The complex geometry of string theory and loop space, in Johns Hopkins Workshop, July 1987 [INSPIRE].

[102] D. Harari, D.K. Hong, P. Ramond and V.G.J. Rodgers, The superstring Diff $S^{1} / S^{1}$ and holomorphic geometry, Nucl. Phys. B 294 (1987) 556 [INSPIRE].

[103] K. Pilch and N.P. Warner, Holomorphic structure of superstring vacua, Class. Quant. Grav. 4 (1987) 1183 [INSPIRE].

[104] T. Hübsch, Calabi-Yau manifolds: a bestiary for physicists, $2^{\text {nd }}$ edition, World Scientific Publishing Co. Inc., River Edge, NJ, U.S.A. (1994) [inSPIRE].

[105] M. Graña, R. Minasian, M. Petrini and D. Waldram, T-duality, generalized geometry and non-geometric backgrounds, JHEP 04 (2009) 075 [arXiv:0807.4527] [INSPIRE].

[106] D. Andriot, M. Larfors, D. Lüst and P. Patalong, A ten-dimensional action for non-geometric fluxes, JHEP 09 (2011) 134 [arXiv:1106.4015] [INSPIRE].

[107] D. Mylonas, P. Schupp and R.J. Szabo, Membrane $\sigma$-models and quantization of non-geometric flux backgrounds, JHEP 09 (2012) 012 [arXiv:1207.0926] [INSPIRE].

[108] D. Andriot, O. Hohm, M. Larfors, D. Lüst and P. Patalong, Non-geometric fluxes in supergravity and double field theory, Fortsch. Phys. 60 (2012) 1150 [arXiv:1204.1979] [INSPIRE]. 
[109] J.L. Bourjaily, A.J. McLeod, M. von Hippel and M. Wilhelm, Bounded collection of Feynman integral Calabi-Yau geometries, Phys. Rev. Lett. 122 (2019) 031601 [arXiv: 1810.07689] [INSPIRE].

[110] J.L. Bourjaily, A.J. McLeod, C. Vergu, M. Volk, M. Von Hippel and M. Wilhelm, Embedding Feynman integral (Calabi-Yau) geometries in weighted projective space, arXiv: 1910.01534 [INSPIRE].

[111] E. Witten, Is supersymmetry really broken?, Int. J. Mod. Phys. A 10 (1995) 1247 [hep-th/9409111] [INSPIRE].

[112] V. Jejjala, R.G. Leigh and D. Minić, The cosmological constant and the deconstruction of gravity, Phys. Lett. B 556 (2003) 71 [hep-th/0212057] [INSPIRE].

[113] K. Becker, M. Becker and A. Strominger, Three-dimensional supergravity and the cosmological constant, Phys. Rev. D 51 (1995) R6603 [hep-th/9502107] [INSPIRE]. 